everything in its way, but the day after the child was bitten, when out with the child's father, it attacked every dog it met, and, among others, bit a cur $\log$ lept at the next house. This dog had a great deal of hair on, and perhaps owing to this cause had, on January 28th, when I caused inquiries to be made, shown no symptoms of rabies. On the same day that the child was bitten, they found the cat dead in the cellar, whether worried or not, they did not know. The doctor advised that the dog should be poisoned, and this was accordingly done.

The child passed urine normally. There was no frothing at the mouth, but the tongue was coated with viscid saliva. In the evening, the same symptoms were apparent, the child going into paroxysms of fright if a cup were brought near him, and yet he could swallow when forced to do so. The pulse was 180 in the minute. The child died early next morning, death appearing to result from exhaustion. There were no convulsions. The period of incubation was thirty days, and the duration of the disease about four days.

The treatment adopted consisted of an aperient powder, containing rhubarb and calomel, which acted upon the bowels, wet sheet packing, and a mixture containing bromide of potassium and tincture of conium in ordinary doses. Ice was ordered, but, I believe, was not obtained.

I have nothing new to offer with regard to the treatment of this affection. Whether large doses of succus conii, or the application of electricity in some form, might allay the painful spasmodic action of the muscles of deglutition, I cannot say. Chloroform and sedative hypodermic injections would, no doubt, be useful in some cases. Whether or not successive transfusions of blood from some animal not subject to hydrophobia, might be of service as a palliative and restorative until the crisis were passed, without experiment it would be rash to speculate, especially as we do not know when such crisis would occur ; indeed, from the invariably fatal result in the human subject, we scarcely know that it would occur at all, except that, by analogy, we may infer that it does, as cases of recovery in the dog are said to take place.

In the ordinary preventive measure of applying caustic to the wound I have little faith. If the dog be not mad, it is a needless cruelty ; and if it be, from what we know respecting the rate of the circulation, and the rapid action of drugs used hypodermically, we may be sure it is of no use if the true skin be penetrated, unless the virus takes its stand temporarily in the tissues around the bite, and requires to undergo development there. If we can, by excising a hard chancre, prevent constitutional syphilis, we may hope similarly to prevent hydrophobia by excision of the wounded part, and the subsequent application of caustic. Very probably, inflamed and suppurative glands are often produced by the free application of caustic to wounds, thus inducing, in debilitating subjects, a cachectic condition, if not so fatal as the disease we hope to prevent, still much worse than what would really have ensued.

\section{A CASE OF APHASIA.}

By E. SCOTT JONES, L.R.C.P., Honorary Medical Officer to the Weston-super-Mare Sanatorium.

Mr. - a gentleman 32 years of age, had always been a hard drinker, and led a very dissipated life. He had occasionally been under my care for bilious attacks, etc., brought on by his indiscriminate way of living.

One afternoon, in the month of August 1870 , he came into my consulting-room, complaining of a pain in his right eye. He evidently had been drinking. I accordingly did not pay much attention, and ordered a mustard plaster behind the ear, and a little aperient medicine.

The next day, I was sent for, and was informed by the wife that she considered her husband very queer; that he had been tipsy the night before, but did not appear to recover. He had slept well. He appeared to me very much the same as I had seen him on former occasions, when under the influence of alcohol; but, on closer examination, I found him suffering from aphasia. On my showing him my watch, and asking him the hour, he told me quite wrong, and, in fact, could not talk sense.

Were I to relate the whole history of this case, I fear it would be tedious to my readers. Suffice it to say, he continued in much the same condition until the commencement of October, sometimes getting better, and then again falling back. He was able to walk out, and looked well, and recognised his friends, but always addressed them by the wrong name. If asked to repeat the alphabet, he invariably mixed the French and English together, and, in fact, presented all the aphasic symptoms. At the commencement of October, he was obliged to take to his bed, and gradually sank into a low typhoid condition, death being ushered in by convulsions.
At the necropsy, all the thoracic and abdominal viscera were healthy, but the brain, although I made the examination only six hours after death, showed general softening ; in fact, the whole of it was in such a state of degeneration, that it was impossible to make any proper examination.

\section{THERAPEUTIC MEMORANDA.}

\section{TREATMENT OF ROUND-WORM.}

IN the JOURNAL of May 23rl, several cases of round-worm are reported. A peculiar case came under my care as an out-patient at the Bristol General Hospital in February last. The patient, a child aged one year and eight months, had been suffering for thrce weeks with a very irritable state of the bowels, so much so that it could not be kept clean; and during an evacuation a round worm was passed. The child looked pale and ill; it had been fed with the ordinary diet of the house and pork. A mixture of a grain of santonine and syrup was ordered to be taken three times a day. On the next visit (four days), the child had voided forty-six round-worms. It seemed more cheerful, and was to continue the mixture. At the next visit, fifteen more had passed, making in all sixty-two worms. Afterwards the child improved rapidly. Thomas E. Clark, M.D., C.M., Clifton.

\section{SURGICAL MEMORANDA.}

\section{TREATMENT OF PALMAR ANEURISM.}

Mr. CRIPPS's interesting, but most troublesome, case of traumatic pal mar aneurism illustrates the serious nature of the disease when treated on the established principle of laying open the sac in order to secure the wounded points. Five cases are mentioned by Mr. Cripps as having been treated in this way; three died of secondary hemorrhage, and two were cured, one of these being Mr. Cripps's own case, in which, after several recurrences of bleeding, the ulnar radial arteries were first tied, but without avail, then the brachial with success. Nine cases were cured by pressure, two others were treated by ligature, and one by injection. I can add another case to the list cured by pressure, which will, perhaps, be of interest in connection with the preceding one. On May Ist, a healthy-looking man, aged 30, was admitted to St. Thomas's Hospital under my care. He had cut the palm of his hand a month previously with a saddler's knife, near the ulnar border. The wound, which was strapped up by a doctor, soon healed, and for three weeks he experienced no trouble. Then the hand began to swell, and he experienced much pain. On admission, a week after, the tumour was of the size of a walnut, occupying the ulnar border of the palm close below the wrist. The skin over the most prominent part was thin and red in colour; the pulsation was very strong, and the tumour verytense. Compression of the arteries at the wrist stopped the pulsation, and the sac could be partially emptied. Subcutaneous injections of ergotin, with instrumental pressure on both radial and ulnar arteries near the wrist, were tried until the following day. No good effect followed; the tumour was larger, more tense, and the skin seemed likely to give way. The forearm was now carefully bandaged with flannel, placed upon a posterior splint, and well elevated upon pillows ; the tumour itself was left uncovered, and an ice-bag laid upon it. Digital pressure was now applied to the brachial artery, the directions given being, that no drop of blood should be allowed to pass. After an interval of forty hours, the aneurism was found to have become harder, and without a trace of pulsation. The following day, it was imagined that slight pulsation could be detected ; this became next morning somewhat more distinct, and was perceptible during the next eleven days; but it was always very faint, and the tumour, nevertheless, continued during the whole time to diminish steadily in size. By way of precaution, digital pressure was ap. plied afresh for twenty-four hours without altering the condition of matters, and the arm was constantly kept upon the splint. On the I6th, a fortnight after the pressure was commenced, it was observed that, during three or four hours at a time, the pulsation wholly disappeared; would then return for a few hours, and again disappear. During this interval until the final disappearance on the evening of the $\mathbf{r} 6 \mathrm{th}$, it was clear that the cause of the pulsation was a supply of blood from the radial artery; pressure on that artery stopped the pulsation; pressure on the ulnar artery produced no effect whatever.

To-day, May 26th, I may pronounce the patient cured. There has been no pulsation in the aneurism during the last ten days. The tumour is being gradually absorbed, and is now about one-third its original bulk. I conclude that the return of pulsation was due to the contraction of 